\title{
Total Laparoscopic Pancreaticoduodenectomy with Left Posterior Superior Mesenteric Artery First- Approach and Plexus-Preserving Circumferential Lymphadenectomy: A Surgical Case Report (With Video)
}

\section{Thanh Khiem NGUYEN}

Bach Mai Hospital

Ham Hoi NGUYEN

Bach Mai Hospital

Tuan Hiep LUONG ( $\sim$ hiep1995hsgs@gmail.com )

Hanoi Medical University

Kim Khue DANG

Bach Mai Hospital

Van Duy LE

Bach Mai Hospital

Yosuke Inoue

Japanese Foundation for Cancer Research

Hong Son TRINH

Viet Duc University Hospital

Duc Dung TRAN

Thai Binh Medical University

\section{Case Report}

Keywords: Total laparoscopic pancreaticoduodenectomy, left-posterior SMA first-approach, SMA plexuspreserving and circumferential lymphadenectomy

Posted Date: February 22nd, 2022

DOI: https://doi.org/10.21203/rs.3.rs-1374836/v1

License: (c) (1) This work is licensed under a Creative Commons Attribution 4.0 International License.

Read Full License 


\section{Abstract}

\section{Introduction:}

Total laparoscopic pancreaticoduodenectomy (tLPD) for cancer of Vater remains a challenging procedure. Recently, several meta-analyses showed the superior aspects of "Superior Mesenteric Artery (SMA)-first approach", "Systematic mesopanreas dissection" and "Circumferential lymphadenectomy around SMA" in increasing R0-resection rate and reducing postoperative complications including pancreatic fistula and bleeding as well as improving overall survival particularly.

\section{Case presentation:}

Our patient is a 70-year-old female with a no special medical history, recruited because of jaundice. She was referred for pancreaticoduodenectomy because of a $10 \mathrm{~mm}$-sized mass in distal bile duct referred to Vater's tumor. We use 5 trocars and the patient placed in a Trendelenburg position. The transverse colon was lifted, the first loop of the jejunum was pulled to the left, the lymph nodes groups 14th and 15th were removed en bloc and then exposed the SMA from the anterior to the left posterior side from the caudal side to the origin. The first jejunal vessels and the posterior inferior pancreaticoduodenal artery were ligated and extensive mobility of the duodenum and head of the pancreas from the left side. The systematic mesopancreas dissection from the right site of the SMA will be easily and conveniently done afterwards. Histopathological examination of ypT2N1 indicated that 1 of the 22 lymph nodes was positive, that was 1 of 7 LNs No. 14. Pathological results showed a Vater adenocarcinoma with all margins being negative.

\section{Conclusions}

This technique was safe and effective to perform precise level-2 mesopancreas dissection and complete lymphadenectomy around SMA without dissection of pl-SMA in laparoscopic field.

\section{Introduction}

Pancreatic head and periampullary tumors, especially pancreatic cancer, still an aggressive lethal disease, with being set to become the second most common cause of cancer-related mortality within the next few years[1]. Pancreaticoduodenectomy (PD) remains the only curative surgical option for pancreatic head and periampullary tumor. In combination with neoadjuvant chemotherapy, the indication of PD is not only in the resectable group, but also extend to group of locally advanced tumors, with up to $60 \%$ of patients with previously unresectable disease became candidates for curative surgery[2]. And with the development of technology and minimally invasive tendency in medicine, minimally invasive pancreaticoduodenectomy, especially total laparoscopic pancreaticoduodenectomy (tLPD) is not out of this way. Recent systematic reviews showed that LPD was superior against open PD in aspects of 
inoperative blood transfusion, wound and pulmonary infection, and shorter hospital stays; and there are no differences of relevant postoperative pancreatic fistula (POPF), severe complications, postoperative mortality, retrieved lymph nodes (LNs), and R0-resection rate[3, 4]. However, to achieve the safety and effectiveness, they also recommended that LPD should be proceed by surgeons with expertise and through learning curve in high-volume centers[3, 4].

With up-to-date knowledges about pathological aspects of oncological process and involvement, there are several new techniques or approaches to make PD becoming complete as an oncologic surgery. And with most discussions and improvements, they are the SMA-first approach, Circumferential lymphadenectomy around SMA with preservation of pl-SMA, and Systematic mesopancreas dissection. Many reports have discussed and shown the superior aspects of these techniques against convenient approach in open LD, but there were few reports taken in tLPD. So that, herein, we reported a technique of tLPD that combined of left posterior SMA-first approach, SMA LNs circumferential as well as systematic mesopanreas (MP) dissection with pl-SMA preserving.

\section{Case Presentation}

The reported case of a 71-year-old female patient without any medical history. She admitted to the hospital because of progressive jaundice, and it lasted in one month but no complain of abdominal discomfort. Her past medical history was unremarkable. There were no significant findings on physical examination with the exception of severe malnutrition. Height was $160 \mathrm{~cm}$ and weight was $50 \mathrm{~kg}$. Laboratory findings were as follows: total bilirubin, direct bilirubin, and albumin was 233.0; $128.0 \mu \mathrm{mol} / \mathrm{I}$ and $3.25 \mathrm{~g} / \mathrm{dL}$ respectively, and amylase was within the normal range. The serum level of carbohydrate antigen (CA)19-9 was $20.2 \mathrm{U} / \mathrm{mL}$, and carcinoembryonic antigen (CEA) was $3.02 \mathrm{ng} / \mathrm{ml}$. Abdominal contrast-enhanced computed tomography (CT) scan revealed a $10 \mathrm{~mm}$ solid hypovascular mass in the periampullary region and distal common bile duct regard to ampullary tumor. The common bile duct and the main pancreatic duct on the distal side of the mass were dilated at $20 \mathrm{~mm}$ and $9 \mathrm{~mm}$ in diameter, respectively. The endoscopic ultrasound (EUS) revealed that a $1.2 \times 1.4 \mathrm{~cm}$ hyperechoic mass in the ampullary of Vater. There was no evidence of lymph node metastasis, peritoneal dissemination, or distant organ metastasis. The upper endoscopy revealed a mass in the duodenal papilla of Vater and the biopsy result was primary duodenal papilla adenocarcinoma. The final diagnosis was an adenocarcinoma tumor of the Ampulla of Vater with TNM Staging was CT1NOMx according to The American Joint Committee on Cancer (AJCC) 8th Staging [5]. MDT meeting consisted of surgeons, physicians, clinical and medical oncologists, radiologists, pathologists, and clinical nurse specialists (CNSs) were organized to make clinical decisions. The informed consent was signed and tLPD following described technique was performed. The operation time was $480 \mathrm{~min}$, the estimated blood loss was $50 \mathrm{ml}$. With no postoperative complications as well as no diarrhea, the patient was discharged on the POD8 uneventfully. Pathological result: intestinal-type ampulla of Vater carcinoma, metastasis to 01/29 lymph node metastasis (LN group

14) was confirmed. The resection margin was negative of tumor involvement (R0).

\section{Surgical Procedure}


Trocars' placement. We use 5 trocars: one $10 \mathrm{~mm}$-trocar placed through the umbilicus for camera; two $12 \mathrm{~mm}$-trocars placed at the midclavicular line higher $1 \mathrm{~cm}$ compared to umbilicus in the right and left side for instrument; two $5 \mathrm{~mm}$-trocars placed at right and left subcostal. Surgeon stands on the right side of patient in the SMA's dissection phase, and changes to the middle position when dissecting the posterior surface of the artery with the rest of the surgical phases, the second and third assistants holding the middle and right cameras, the first assistant standing on the side left.

\section{Step 1: Mobilize the first jejunal loop, approach the superior mesenteric artery (SMA) from left-posterior}

side. After exploring the peritoneum to exclude metastases, the right-sided assistant lifts the mesentery of transverse colon upward, and the left-sided assistant pulls the first jejunum to the left side. We open the peritoneal along meso of the first jejunal loop, dissecting the lymph nodes group 14, group 15 along the first jejunal artery (FJA), middle colic artery and right colic artery. Exposing the FJA adjacent to the superior mesenteric artery (SMA), isolate and ligate close to the origin of the FJA. Dissection the left border of the origin of SMA just above the left renal vein (LRV), including LN station No. 14 or left-sided SMA LNs (Video 1).

After transecting the first jejunal loop with GI stapler with corresponding mesenteric, the Ligament of Treitz is then mobilized with dissection of the 3rd and 4th portions of the duodenum. Assess and classify the anatomy of the first jejunal vein (FJV) before and during surgery. In case on the anterior, simple approach and dissection. Most of the cases we found the common trunk between the FJV, and the inferior pancreaticoduodenal vein (IPDV) goes posteriorly to the SMA: after ligation of the FJA, the SMA was dissected and lifted, approached the FJV, and hemo-locked just below the left side of the SMA, then continue dissecting the first jejunal loop and the pancreatic uncinate process behind this vein.

The main surgeon changes to middle position. We continue dissecting on the posterior side of the SMA, ligate the posterior pancreaticoduodenal artery (PPDA) (if any) on this posterior surface. Dissecting the superior border and then passing the superior border to the right border to separate the SMA from the SMV. The neural plexus around the SMA is carefully preserved. (Figure 1). We found that extensive mobilization of the duodenum and head of the pancreas from the left side (Kocher maneuver) to the proximal right peritoneal fold of the duodenum is easy during this phase.

\section{Step 2: Mobilization of the entire pancreaticoduodenal mass and approach to the superior mesenteric vein from posterior side of pancreas. Detach the great omentum from transverse colon, detach hepatic flexure from the duodenum and pancreatic head to the lower border of the pancreas. Exposing the right margin of the superior mesenteric vein (SMV) and dissect the gastrocolic trunk of Henle (the confluence of the veins of the pancreatic head and the right colon before flow into the SMV). Mobilizing the hepatic flexure of the colon and the duodenum. Expose the posterior surface of the duodenum, the head of the pancreas to the left border of the aorta above the renal vein at the origin of the SMA.}

Step 3: Transect the stomach and pancreatic neck; dissect the hepatoduodenal ligament and the celiac lymph nodes. We start dissecting of right gastroepiploic artery (RGEA) right gastric artery from the level of the antrum. The stomach was transected using a stapler about $2 \mathrm{~cm}$ from the pylorus. The right gastric 
artery (RGA) is transected at its origin, then we dissect the lymph nodes groups $8 a, 12 a, 7,9$ in front of the hepatoduodenal ligament to the origin of left gastric artery and superior border of celiac trunk. The gastroduodenal artery (GDA) is dissected and ligated and isolating the common hepatic artery (CHA) from the origin to the GDA's bifurcation. The tissue plane between the inferior surface of the pancreatic neck is then blunt dissected and reveal the anterior surface of the SMV and the portal vein (PV). A nelaton is inserted from the inferior to superior side of the pancreas. We transect the pancreas at the pancreas neck and expose the pancreatic duct with at least $5 \mathrm{~mm}$ in length. After resection of the pancreatic neck, we carry on dissecting of lymph nodes groups $8 b, 12 p$ in the inferior surface of the PV and right-sided celiac artery $(\mathrm{CA})$.

Step 4: Isolate the entire pancreaticoduodenal mass from the SMA, SMV, and PV. Proximal loop of jejunum is passed to the right side through retroperitoneal defect. We systematically perform dissecting the meso-pancreas from SMV, SMA and PV. Resect the entire mesopancreas both anterior and posterior sides of SMA including right-sided SMA LNs (Video 2). This procedure is often quite difficult with the convenient technique but becomes much easier with Left Posterior SMA-first Approach. Continue dissecting the lymph nodes posterior to the main bile duct up to the proximal side of hepatic hilum. This step was proceeded easily with our technique after the entire pancreaticoduodenal mass mobilize subtotally after this step. After the cholecystectomy, the common bile duct was dissected and divided at the level of right hepatic artery. Specimens were taken en bloc: duodenal and pancreatic head, lymph nodes group 5, 6, 7, 8, 9, 12a, 12b1, 12b2, 12v, 13, 14p, 14d (or left-sided SMA LNs), the right-sided SMA LNs and 15.

\section{Step 5: Small opening at umbilicus, remove the sample.}

Step 6: Reconstruction phase. The first loop of the jejunum is then brought through the transverse mesocolon. Pancreatojejunostomy is performed with double layers end -to-side, modified-Blumgart fashion. Inner layer is duct to mucosa anastomosis, using 5-0 monosyl suture (6 stitches: 3 posterior, 3 anterior). We place an internal drainage with a plastic stent. Second layer is 2 interrupted $U$ shape suturing between posterior and anterior pancreatic walls and seromuscular layer of the jejunum using Prolene 3/0. Hepaticojejunostomy is performed distal to the pancreatojejunostomy about $10 \mathrm{~cm}$ with one layer fashion. The anastomosis of the common hepatic duct to the jejunum in an end-to-side fashion using 5-0 monosyl continuous suture for the posterior layer and interrupted suture for the anterior layer. A distal loop of jejunum approximately $60 \mathrm{~cm}$ distal to defect in the transverse mesocolon is brought antecolic. Antecolic gastrojejunostomy performed via the enterotomy and gastrotomy using a GI stapler. Defect in gastrojejunostomy is oversewn with interrupted 3-0 Vicryl suture (Video 3).

\section{Discussion And Conclusion}

The history of the concept arteries-first approach begun in 1993, when Nakao et al. first represented a technique of "isolated pancreatectomy using catheter bypass of the portal vein" for pancreatic head carcinoma, while all arteries and drainage veins that supply the pancreatic head region are ligated and 
divided [6]. After that, the concept "SMA-first approach" was firstly introduced by Patrick Pessaux et al. in a technical article in 2006 [7]. This technique started from some reasons: firstly, the requirement of accurate preoperative stage and resectability of the tumor following guidelines of the National Comprehensive Cancer Network (NCCN) [8], and secondly, high rate of postoperative morbidity and mortality strongly related to the complexity of anatomical variation of superior mesenteric vessel branches and tributaries. In concept, the term "SMA-first approach" means exploration of superior mesenteric vessels as well as celiac trunk and portal vein to detect the state of tumoral invasion of these vessels and determine the resectable conditions before the point of no return (the step of pancreatic neck's dissection or bile duct division) [7, 9]. Until now, many methods have been developed to achieve the "SMA-first approach" concept in open PD, and according to our knowledge, there are totally six methods that have been summarized by Sanjay, $\mathrm{P}$ et al. with advantages, disadvantages and indications of each approaches[10]. Recently, several meta-analyses showed the superior aspects of "SMA-first approach" in R0-resection rate, postoperative complications including pancreatic fistula and bleeding as well as overall survival[11, 12]. In a recent systematic review by Nagakawa Y. et al, with laparoscopic PD, methods for the "SMA-first approach" concept were modified based on these approaches in open PD, and categorized into four approaches included: anterior approach, posterior approach, right approach, and left approach[13]. In posterior approach, the posterior side of the SMA was exposed above the LRV from the right side[14] or from the caudal side[15]. In left approach, Cho et al. described a surgical technique to divide the common artery of the IPDA and the FJA by pulling the proximal jejunum to the right to visualize the ligament of Treitz and the origin of the SMA is visualized just above the LRV[16]. In our technique, we divide the FJA or the common artery of the IPDA and the FJA after removal of lymph nodes group 15 and circumferential SMA lymph nodes on the left anterior side to the posterior side by pulling the proximal jejunum to the left (Figure 3), then after dissecting the first jejunal loop, the SMA was exposed from the left and caudal side. So that, we call our technique "the left-posterior first approach".

Beside "SMA-first approach", total or systematic mesopanreas (MP) dissection also a concept that has been paid a lot of attention recently[17]. Started by Gockel et al. in 2007[18], in definition, mesopancreas was a retropancreatic well-vascularized and nerve-rich structure surrounding the SMA, including the first and second nerve plexuses of the pancreas head (pIPh-I and pIPh-II) according to the new classification of Japan Pancreas Society as well as some landmarks: inferior pancreatoduodenal arteries (IPDAs), jejunal arteries (JAs), jejunal veins (JVs); and lymph nodes (LNs)[19]. In anatomical aspect, mesopanreas connects the pancreatic head to the SMA and right celiac ganglion[17]. Many retrospective studies were proceeded and summarized in a systematic review by J. M. Ramia et al. in 2018 with conclusions of MP excision increased the R0-resection rate, so that improved the oncological outcomes[20]. According to Inoue et al, there are 3 levels of the systematic dissection of mesopancreas, which were applied due to type and extent of pancreatic head tumors, and Level-2 systematic MP dissection includes en bloc LNs dissection in the MP by the central vessel ligation technique, which is implemented to ampullary/lower bile duct/duodenal cancer or selective invasive pancreatic ductal cancer[17]. In our technique, the mesopancreas was resected systematically en bloc with entire pancreaticoduodenal mass. The mesopancreas size was recorded in this case and all other PD cases we proceeded (Figure 4A, B). 
One of the most impact factors that influent the survival outcomes of resectable pancreatic ductal adenocarcinoma (PDAC) is high frequency of lymph node metastasis, especially the left side of LNs around SMA, due to right-sided soft tissues including LNs are usually resected in conventional PD [21, 22]. Following new Japanese $L N$ station system, the $L N$ No.14 divided to $L N$ No.14p and $14 d$, and located in the left side of the SMA (left-side LNs of SMA), and located in an anatomical-surgical layer of the "mesopancreatoduodenum", which was along the IPDA and the FJA [23, 24]. So that, systematic MP dissection do not include left-side SMA LNs. Otherwise, the rate of left LNs No.14 metastasis according to Okada's study was $12 \%$ [25]. There were some preliminary evidences proved the superior survival outcomes of SMA LNs circumferential dissection, and not increased the rate of postoperative complications especially postoperative diarrhea [21]. And in another aspect, the concept of right-half dissection of the SMA nerve plexus along with SMA LNs circumferential dissection, in order to accomplish R0 resection with the potential for nerve plexus invasion, showed no significant difference in oncological outcome, as well as significantly increased the rate of postoperative diarrhea requiring opioids according to recent studies and trials [26, 27].

\section{Conclusion}

Herein, we reported a successfully first-describe total laparoscopic left posterior first-approach and superior mesenteric artery plexus-preserving pancreaticoduodenectomy with circumferential dissection of lymph nodes in a patient of primary duodenal papilla adenocarcinoma with Staging pT2N1M0 with negative margin (R0 resection). There were no short-term complications. We think this technique was safe and effective to perform precise level-2 dissection in laparoscopic field. Further investigations and follow-up must be proceeded to evaluate the long-term outcomes of our technique.

\section{Abbreviations}

AJCC American Joint Committee on Cancer

CA Celiac artery

CEA Carcinoembryonic antigen

CHA Common hepatic artery

CT Computed tomography

EUS Endoscopic ultrasound

FJA First jejunal artery

FJV First jejunal vein

GDA Gastroduodenal artery 
IPDA Inferior pancreaticoduodenal artery

IPDV Inferior pancreaticoduodenal vein

LN Lymph nodes

LRV Left renal vein

NCCN National Comprehensive Cancer Network

PDAC Pancreatic ductal adenocarcinoma

POPF Postoperative pancreatic fistula

PPDA Posterior pancreaticoduodenal artery

LPD Laparoscopic pancreaticoduodenectomy

SMA Superior mesenteric artery

SMV Superior mesenteric vein

RGA Right gastric artery

\section{Declarations}

\section{Funding}

The authors declare no funding for this study.

\section{Funding}

The authors declare no funding for this study.

\section{Conflict of interests}

The authors declare that they have no conflicts of interests.

\section{Ethics approval}

The study was approved by the Research Ethics Committee of Bach Mai Hospital. The procedures used in this study adhere to the tenets of the Declarations of Helsinki.

\section{Consent to participate.}

The patients have consented to the submission of the case report for submission to the journal. 


\section{Consent to publication}

Not applicable

\section{Availability of data and material}

Data is available upon reasonable request and with permission of Bach Mai Hospital.

\section{Acknowledgements}

Thanks to all colleagues in Department of Gastrointestinal and Hepato-pancreato-biliary surgery, Bach Mai Hospital for helping us in taking care of patients.

\section{Author's contributions}

Thanh Khiem NGUYEN: the main doctor conceived the original idea and operated the patients, wrote manuscript.

Ham Hoi NGUYEN: the main doctor conceived the original idea and operated the patients, wrote manuscript.

Tuan Hiep LUONG: followed up the patients, wrote manuscript, designed technical videos and figures.

Kim Khue DANG: operated the patients, summed up, revised manuscript, designed technical videos and figures.

Van Duy LE: operated the patients, summed up, revised manuscript.

Yosuke INOUE: the supervisor conceived the original idea, summed up, revised manuscript.

Hong Son TRINH: summed up, revised manuscript.

Duc Dung TRAN: designed technical videos and figures.

All authors contributed to the interpretation of the results, discussed the results. All authors read and approved the final manuscript to submit.

\section{References}

1. Rahib L, Smith BD, Aizenberg R, Rosenzweig AB, Fleshman JM, Matrisian LM: Projecting cancer incidence and deaths to 2030: the unexpected burden of thyroid, liver, and pancreas cancers in the United States. Cancer research 2014, 74(11):2913-2921.

2. Hackert T, Sachsenmaier M, Hinz U, Schneider L, Michalski CW, Springfeld C, Strobel O, Jäger D, Ulrich A, Büchler MW: Locally Advanced Pancreatic Cancer: Neoadjuvant Therapy With Folfirinox Results in Resectability in $60 \%$ of the Patients. Annals of surgery 2016, 264(3):457-463. 
3. Aiolfı A, Lombardo F, Bonitta G, Danelli P, Bona D: Systematic review and updated network metaanalysis comparing open, laparoscopic, and robotic pancreaticoduodenectomy. Updates in surgery 2021, 73(3):909-922.

4. Feng Q, Xin Z, Qiu J, Xu M: Laparoscopic vs. Open Pancreaticoduodenectomy After Learning Curve: A Systematic Review and Meta-Analysis of Single-Center Studies. Frontiers in surgery 2021, 8:715083.

5. Amin M, Edge S: ES, Greene F. AJCC Cancer Staging Manual. In.: Chicago, IL: Springer; 2017.

6. Nakao A, Takagi $\mathrm{H}$ : Isolated pancreatectomy for pancreatic head carcinoma using catheter bypass of the portal vein. Hepato-gastroenterology 1993, 40(5):426-429.

7. Pessaux P, Varma D, Arnaud JP: Pancreaticoduodenectomy: superior mesenteric artery first approach. Journal of gastrointestinal surgery: official journal of the Society for Surgery of the Alimentary Tract 2006, 10(4):607-611.

8. Tempero MA, Malafa MP, Al-Hawary M, Behrman SW, Benson AB, Cardin DB, Chiorean EG, Chung V, Czito B, Chiaro MD et al: Pancreatic Adenocarcinoma, Version 2.2021, NCCN Clinical Practice Guidelines in Oncology. Journal of the National Comprehensive Cancer Network 2021, 19(4):439457.

9. Weitz J, Rahbari N, Koch M, Büchler MW: The "Artery First" Approach for Resection of Pancreatic Head Cancer. Journal of the American College of Surgeons 2010, 210(2):e1-e4.

10. Sanjay P, Takaori K, Govil S, Shrikhande SV, Windsor JA: 'Artery-first' approaches to pancreatoduodenectomy. The British journal of surgery 2012, 99(8):1027-1035.

11. Ironside N, Barreto SG, Loveday B, Shrikhande SV, Windsor JA, Pandanaboyana S: Meta-analysis of an artery-first approach versus standard pancreatoduodenectomy on perioperative outcomes and survival. British Journal of Surgery 2018, 105(6):628-636.

12. Jiang X, Yu Z, Ma Z, Deng H, Ren W, Shi W, Jiao Z: Superior mesenteric artery first approach can improve the clinical outcomes of pancreaticoduodenectomy: A meta-analysis. International Journal of Surgery 2020, 73:14-24.

13. Nagakawa Y, Watanabe Y, Kozono S, Boggi U, Palanivelu C, Liu R, Wang SE, He J, Nishino H, Ohtsuka $\mathrm{T}$ et al: Surgical approaches to the superior mesenteric artery during minimally invasive pancreaticoduodenectomy: A systematic review. Journal of hepato-biliary-pancreatic sciences 2021.

14. Wang XM, Sun WD, Hu MH, Wang GN, Jiang YQ, Fang XS, Han M: Inferoposterior duodenal approach for laparoscopic pancreaticoduodenectomy. World journal of gastroenterology 2016, 22(6):21422148.

15. Honda G, Kurata M, Okuda Y, Kobayashi S, Sakamoto K, Takahashi K: Laparoscopic Pancreaticoduodenectomy: Taking Advantage of the Unique View from the Caudal Side. Journal of the American College of Surgeons 2013, 217(6):e45-e49.

16. Cho A, Yamamoto H, Kainuma O: Tips of laparoscopic pancreaticoduodenectomy: superior mesenteric artery first approach (with video). Journal of hepato-biliary-pancreatic sciences 2014, 21(3):E19-21. 
17. Inoue Y, Saiura A, Yoshioka R, Ono Y, Takahashi M, Arita J, Takahashi Y, Koga R:

Pancreatoduodenectomy With Systematic Mesopancreas Dissection Using a Supracolic Anterior Artery-first Approach. Annals of surgery 2015, 262(6):1092-1101.

18. Gockel I, Domeyer M, Wolloscheck T, Konerding MA, Junginger T: Resection of the mesopancreas (RMP): a new surgical classification of a known anatomical space. World journal of surgical oncology 2007, 5:44.

19. Isaji S, Murata Y, Kishiwada M: New Japanese Classification of Pancreatic Cancer. In: Pancreatic Cancer. edn. Edited by Neoptolemos JP, Urrutia R, Abbruzzese JL, Büchler MW. New York, NY: Springer New York; 2018: 1021-1037.

20. Ramia JM, De-la-Plaza R, Manuel-Vazquez A, Lopez-Marcano A, Morales R: Systematic review of the mesopancreas: concept and clinical implications. Clinical and Translational Oncology 2018, 20(11):1385-1391.

21. Kondo N, Uemura K, Nakagawa N, Okada K, Seo S, Takahashi S, Murakami Y: Superior Mesenteric Artery Plexus-Preserving Pancreatoduodenectomy with Circumferential Dissection of Lymph Nodes. Journal of gastrointestinal surgery: official journal of the Society for Surgery of the Alimentary Tract 2020, 24(7):1712-1719.

22. Kim JR, Kim H, Kwon W, Jang JY, Kim SW: Pattern of local recurrence after curative resection in pancreatic ductal adenocarcinoma according to the initial location of the tumor. Journal of hepatobiliary-pancreatic sciences 2021, 28(1):105-114.

23. Kawabata $Y$, Hayashi $H$, Ishikawa $N$, Tajima Y: Total meso-pancreatoduodenum excision with pancreaticoduodenectomy in lower biliary tract cancer. Langenbeck's archives of surgery 2016, 401(4):463-469.

24. Kang MJ, Kim S-W: En bloc proximal peri-mesenteric clearance for pancreatic head cancer surgery. Ann Hepatobiliary Pancreat Surg 2020, 24(4):389-395.

25. Okada K, Murakami Y, Kondo N, Uemura K, Nakagawa N, Seo S, Takahashi S, Sueda T: Prognostic Significance of Lymph Node Metastasis and Micrometastasis Along the Left Side of Superior Mesenteric Artery in Pancreatic Head Cancer. Journal of gastrointestinal surgery: official journal of the Society for Surgery of the Alimentary Tract 2019, 23(10):2100-2109.

26. Inoue Y, Saiura A, Oba A, Kawakatsu S, Ono Y, Sato T, Mise Y, Ishizawa T, Takahashi Y, Ito H: Optimal Extent of Superior Mesenteric Artery Dissection during Pancreaticoduodenectomy for Pancreatic Cancer: Balancing Surgical and Oncological Safety. Journal of gastrointestinal surgery: official journal of the Society for Surgery of the Alimentary Tract 2019, 23(7):1373-1383.

27. Yamada S, Satoi S, Takami H, Yamamoto T, Yoshioka I, Sonohara F, Yamaki S, Shibuya K, Hayashi M, Hashimoto $\mathrm{D}$ et al: Multicenter randomized phase II trial of prophylactic right-half dissection of superior mesenteric artery nerve plexus in pancreatoduodenectomy for pancreatic head cancer. Annals of gastroenterological surgery 2021, 5(1):111-118.

\section{Figures}



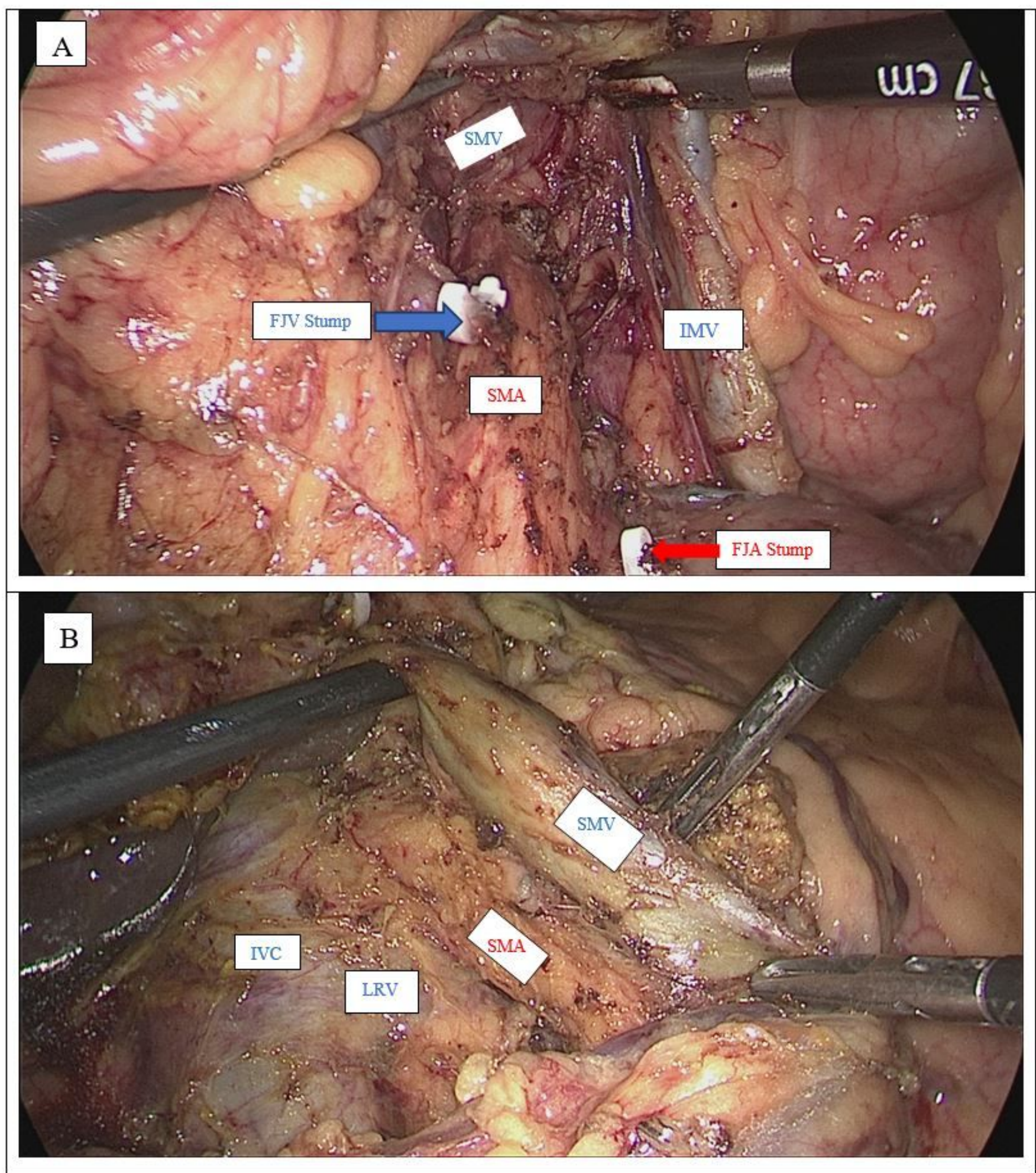

\section{Figure 1}

Preservation of the neural plexus around the SMA (A. Left side; B. Right side)

(SMA. Superior mesenteric artery, SMV. Superior mesenteric vein, IMV. Inferior mesenteric vein, PV. Portal vein, IVC. Inferior vena cava, FJA: First jejunum artery, FJV: First jejunum vein, LRV: Left renal vein) 


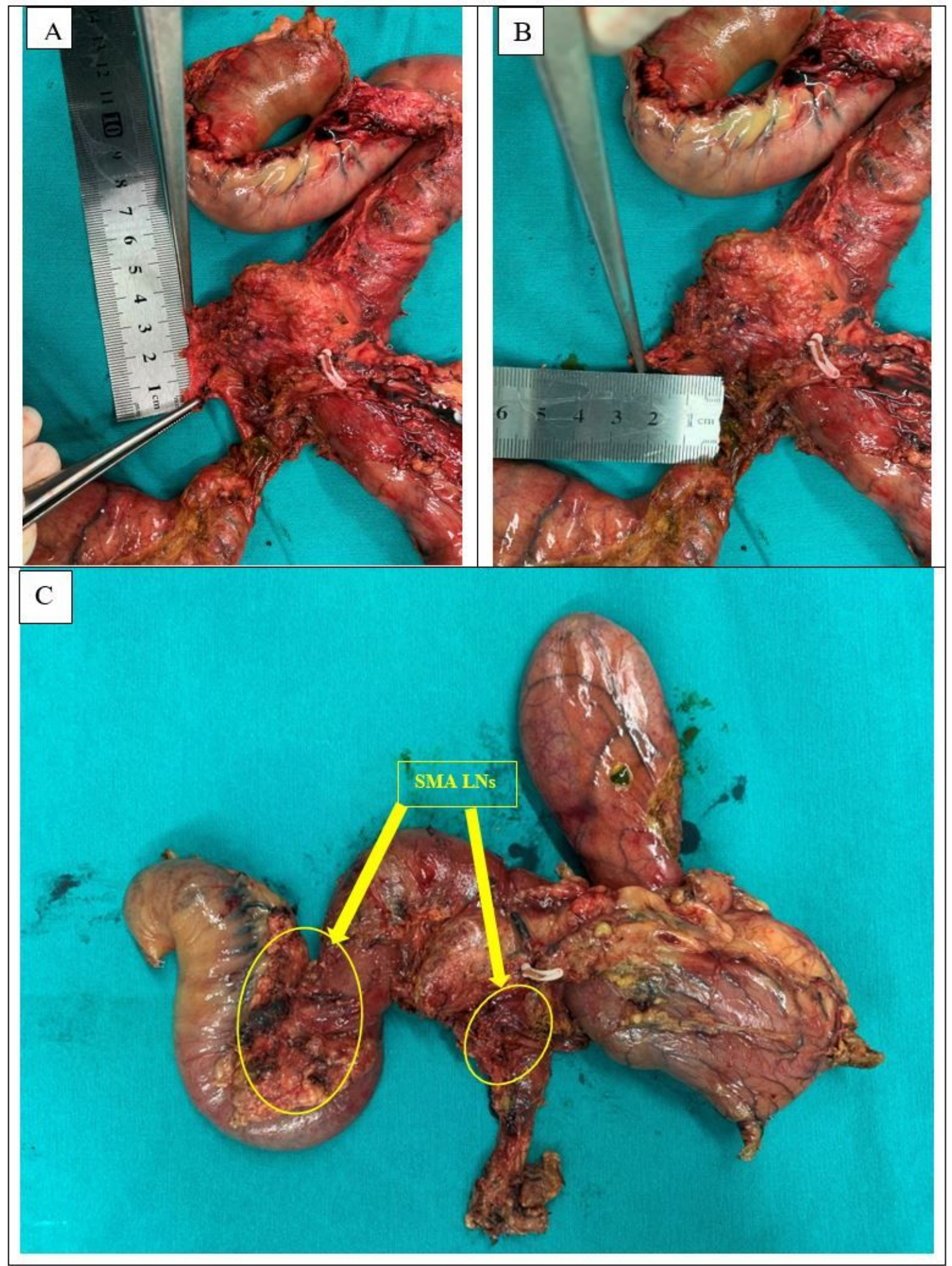

Figure 2

Specimens.

A and B. The mesopancreas size was recorded systematically in our protocol 
C. SMA LNs including right-side and left-side en bloc

\section{Supplementary Files}

This is a list of supplementary files associated with this preprint. Click to download.

- Video1.mp4

- Video2.mp4

- Video3.mp4 